\title{
Implications for Farnesoid X Receptor Signaling on Bile Acid Metabolism as a Potential Therapeutic Strategy for Nonalcoholic Fatty Liver Disease
}

\author{
Hyekyung Yang ${ }^{1}$, Cheol-Young Park ${ }^{1,2, *}$ \\ ${ }^{1}$ Medical Research Institute, Kangbuk Samsung Hospital, Sungkyunkwan University School of Medicine, Seoul; ' 2 Division of Endocrinology and Metabolism, \\ Department of Internal Medicine, Kangbuk Samsung Hospital, Sungkyunkwan University School of Medicine, Seoul, Korea
}

Non-alcoholic fatty liver disease (NAFLD) is the most common chronic liver disease in both developed and developing countries and is an important risk factor for both hepatic and cardiometabolic mortality. Despite decades of clinical trials, effective treatment options for NAFLD are limited, requiring novel therapeutic approaches to prevent disease development and progression to cirrhosis and cancer. Recently, bile acids have emerged as signaling molecules and metabolic regulators that can activate signaling mediated by nuclear receptors and $\mathrm{G}$ protein-coupled receptors to regulate hepatic lipid, glucose, and energy homeostasis, as well as its own synthesis and transport in the liver and intestine. Many recent studies have reported that the activation or modulation of bile acid signaling mediated by bile acid receptors favorably affects both insulin sensitivity and NAFLD pathogenesis at multiple levels, suggesting that these approaches hold promise as novel therapies. In this review, we provide an overview of the role of bile acids, in particular, their signaling related to the nuclear receptor farnesoid X receptor in NAFLD and new insights into the possible approach of targeting bile acid-related pathways in the treatment of this serious disease.

Key words: Bile acids, Farnesoid X receptor, Farnesoid X receptor agonists, Gut microbiota, Non-alcoholic fatty liver disease

\section{Introduction}

Non-alcoholic fatty liver disease (NAFLD) is characterized by massive ectopic triglyceride accumulation in the liver in the absence of other chronic liver disease or significant alcohol consumption. ${ }^{1}$ NAFLD is the most common chronic liver disease in developed and developing countries, affecting $20-30 \%$ of the adult population and more than $80 \%$ of obese individuals around the world. ${ }^{2}$ NAFLD refers to a wide spectrum of liver disorders, ranging from simple fatty liver (steatosis) to nonalcoholic steatohepatitis (NASH) and liver cirrhosis. NAFLD begins with simple steatosis, which can progress to $\mathrm{NASH}$, which involves hepatic steatosis with inflammation, fibrosis, and hepatocyte injury. NASH may further progress to liver cirrhosis, eventually leading to hepatocellular carcinoma and liver failure. ${ }^{1}$ In addition, NAFLD is strongly associated with insulin resistance, type 2 diabetes, coronary heart disease, and atherosclerosis. ${ }^{2}$

To date, treatment options for NAFLD are limited and are direct- ed primarily at weight loss, lifestyle modifications, or pharmacological improvement of the risk factors that are commonly associated with metabolic syndrome. ${ }^{1}$ Although no pharmacological treatment has currently been approved for NAFLD/NASH, therapies with insulin sensitizers, such as pioglitazone and metformin, and antioxidants such as vitamin $\mathrm{E}$ are among the best-established approaches. ${ }^{1}$ However, these approaches have conservative efficacy (pioglitazone and metformin in patients with type 2 diabetes and vitamin $\mathrm{E}$ in non-diabetic patients) and long-term safety issues (vitamin E treatments have increased mortality, and pioglitazone is associated with various adverse effects, including weight gain, fluid retention, and osteoporosis), complicating their clinical use. ${ }^{1}$ In this review, we provide an overview of the role of bile acids and their signaling in NAFLD and provide new insights into the possible approach of targeting bile acid-related pathways in the treatment of this serious global health problem.
*Corresponding author Cheol-Young Park (iD http://orcid.org/0000-0002-9415-9965 Medical Research Institute \& Division of Endocrinology and Metabolism, Department of Internal Medicine, Kangbuk Samsung Hospital, Sungkyunkwan University School of Medicine, 29 Saemunan-ro, Jongno-gu, Seoul 03181, Korea

Tel +82-2-2001-1869 Fax +82-2-2001-1588 E-mail cydoctor@chol.com
Copyright (C) 2016 Korean Society for the Study of Obesity

(a) This is an Open Access article distributed under the terms of the Creative Commons Attribution Non-Commercial License (http://creativecommons.org/licenses/by-nc/4.0/) which permits unrestricted non-commercial use, distribution, and reproduction in any medium, provided the original work is properly cited. 


\section{Bile acids}

Bile acid synthesis is the major pathway for cholesterol catabolism and accounts for $\sim 50 \%$ of the daily turnover of cholesterol. ${ }^{3,4}$ The synthesis of bile acids occurs exclusively in the liver, which converts hydrophobic cholesterol into more water-soluble amphiphatic compounds. ${ }^{4}$ Cholic acid (CA, $\left.3 \alpha, 7 \alpha, 12 \alpha-\mathrm{OH}\right)$ and chenodeoxycholic acid (CDCA, $3 \alpha, 7 \alpha-\mathrm{OH})$ are the major primary bile acids in humans. ${ }^{4}$ They are conjugated with taurine or glycine for secretion into bile and are stored in the gallbladder. After meals, bile acids are released into the gastrointestinal tract. In the intestine, conjugated bile acids are first de-conjugated, and then gut bacterial $7 \alpha$-hydroxylase converts CA and CDCA to deoxycholic acid (DCA, 3a,12a) and lithocholic acid (LCA: $3 \alpha$ ), respectively, two major secondary bile acids in humans. ${ }^{4}$ Most bile acids (95\%) are reabsorbed in the intestine and transported back to the liver (enterohepatic circulation); only $\sim 5 \%$ of bile acids are not reabsorbed and are eliminated in the feces. ${ }^{4}$ Enterohepatic circulation maintains a bile acid pool size of approximately $4 \mathrm{mg}$ in mice and 2 to $4 \mathrm{~g}$ in humans. ${ }^{4}$ Bile acid loss by fecal bile acid excretion is replenished via de novo synthesis (i.e., conversion of cholesterol to bile acids) in the liver to maintain a constant bile acid pool; this process represents a major route for elimination of cholesterol 6 from the body. ${ }^{5}$

The bile acid synthesis is tightly regulated by a negative feedback mechanism within the liver and intestine (Fig. 1). In the liver, cholesterol 7 $\alpha$-hydroxylase (CYP7A1) is the first and rate-limiting enzyme of bile acid biosynthesis to produce CA and CDCA from cholesterol in humans. ${ }^{6}$ Bile acid returning to the liver via enterohepatic circulation may directly or indirectly inhibit bile acid synthesis by inhibiting CYP7A1 activity. Interruption of enterohepatic circulation of bile acids by bile acid sequestrants such as cholestyramine strongly stimulated CYP7A1 enzyme activity and bile acid synthesis in human

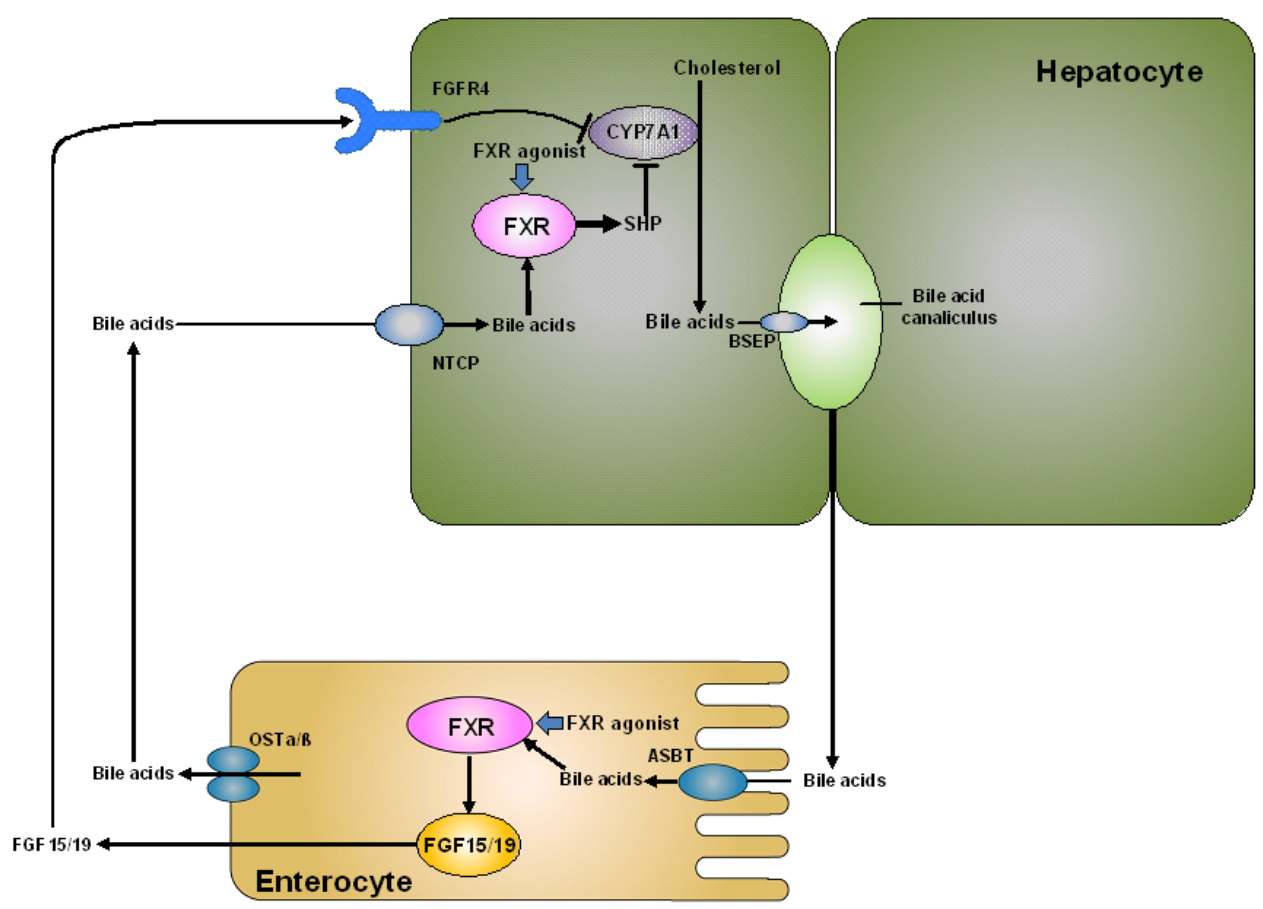

Figure 1. Synthesis, transport, and enterohepatic circulation of bile acids and roles of FXR. Bile acids are produced in the liver by CYP7A1-initiated conversion of cholesterol to primary bile acids. Bile acids are secreted via BSEP into biliary canalicular lumen. In the ileum, bile acids are reabsorbed via ASBT in terminal ileum enterocytes. Here, they bind and activate FXR, which stimulates production and secretion of FGF15 (mouse)/FGF19 (human) into the portal circulation. In the liver, FGF15/FGF19 binds to its receptor FGFR4, which activates a signaling pathway involving MAP kinases and causes repression of CYP7A1 transcription, thus downregulating bile acid synthesis. After OST $\alpha / \beta$-mediated secretion into the portal circulation, bile acids are taken up by the liver via NTCP, completing the enterohepatic cycle. In the liver, bile acids bind to FXR, which upregulate SHP to interfere with the expression of CYP7A1. FXR agonists may affect FXR in both the liver and intestine, and this strongly downregulates CYP7A1, both by FGF15/19-dependent and -independent pathways.

ASBT, apical sodium-dependent bile salt transporter; BSEP, bile salt export pump; CYP7A1, cholesterol 7 $\alpha$-hydroxylase; FGF15/FGF19, fibroblast growth factor 15/fibroblast growth factor 19; FGFR4, fibroblast growth factor receptor 4; FXR, farnesoid X receptor; NTCP, Na+-dependent taurocholate cotransport peptide; SHP, small heterodimer partner.

Figure and caption reprinted from Schaap et al. Nat Rev Gastroenterol Hepatol (2014)12 after modification with permission. 
and mice. ${ }^{7}$ In contrast, treatment with CDCA suppressed CYP7A1 activity and bile acid synthesis. ${ }^{7,8}$

For many years, the roles of bile acids have been thought to be stimulating hepatic bile flow by canalicular bile salt secretion, aiding pancreatic lipase to digest emulsified fat into monoglycerides and fatty acids, which can be absorbed in the proximal small intestine, and solubilizing the cholesterol that can help intestinal absorption and excretion in the feces. ${ }^{4}$ Therefore, bile acid synthesis is considered as the principle means of removal of cholesterol from the body. However, in the 1990s, a number of nuclear hormone receptors, which are intracellular transcription factors activated by ligand, were discovered; later, bile acids were identified as their endogenous ligands. ${ }^{9-11}$ The nuclear receptors for which bile acids are relevant ligands are farnesoid $\mathrm{X}$ receptor $(\mathrm{FXR}, \mathrm{NR} 1 \mathrm{H} 4)$, vitamin $\mathrm{D}_{3}$ receptor (VDR, NR1I1), and pregnane X receptor (PXR, NR1I2). ${ }^{12}$ Recently, G-protein-coupled receptors, TGR5 and sphingosine-1-phosphate receptor 2 (S1PR2) have been identified as a bile acid-activated membrane receptors. ${ }^{12}$ These findings led to the ideas that bile acids may function as signaling molecules that regulate other metabolic processes, such as glucose, lipid, and energy homeostasis, as well as their own synthesis and metabolism, mediated by these receptors. ${ }^{4}$ Herein, our review will focus on the role of FXR in lipid and glucose metabolism and inflammation, particularly associated with the etiology of NAFLD and NASH.

\section{Role of FXR on bile acid signaling}

FXR was originally cloned as an "orphan" receptor in $1995 \cdot{ }^{13,14}$ In 1999, three groups identified bile acids as the endogenous ligands for FXR; shortly thereafter, bile acids were found to regulate their own synthesis by an FXR-mediated feedback mechanism (Fig. 1). ${ }^{9-11,15}$ FXR is highly expressed in the liver and intestine, which are the tissues engaged in the enterohepatic circulation of bile acids, and to a lesser extent in the kidney, adrenal gland, and vascular systems. ${ }^{12}$ FXR can be activated by both primary and secondary conjugated bile acids; CDCA is the most potent bile acid ligand of FXR $\left(\mathrm{EC}_{50}=\right.$ $\sim 10 \mu \mathrm{mol} / \mathrm{L})$, followed by LCA, DCA, and CA. Ursodeoxycholic (UDCA) and muricholic acids (MCAs) cannot activate FXR. ${ }^{9,10}$ In the liver, bile acid-activated FXR induces a negative receptor small heterodimer partner (SHP) to inhibit CYP7A1 transcription by repressing hepatic nuclear factor $4 \alpha$ (HNF4a) and liver receptor ho- mologue-1 (LRH-1), which bind to the bile acid response element in the CYP7A1 gene promoter. ${ }^{4}$ In the intestine, reabsorbed bile acids also activate FXR, subsequently stimulating secretion of fibroblast growth factor 15 (FGF15, FGF19 in humans) that travels to the liver and activates hepatic FGF receptor 4 (FGFR4) signaling to inhibit CYP7A1 and sterol-12 $\alpha$-hydroxylase (CYP8B1) gene expressions and bile acid synthesis. ${ }^{4}$ In addition, FXR is known to critically regulate biliary bile acid secretion, intestinal bile acid reabsorption and secretion, and hepatic uptake of bile acid via bile acid transporters, as well as bile acid conjugation. ${ }^{12,16}$ FXR regulates bile acid conjugation by inducing gene transcriptions of conjugation enzymes bile acidCoA:amino $\mathrm{N}$-acetyltransferase (BAAT) and bile acid:CoA synthase (BACS). ${ }^{6}$ FXR also induces canalicular transporters; bile salt export pump (BSEP, a ATP binding cassette transporter, ABCB11) for secretion of the major bile acid species; a multidrug resistant protein $2 / 3$ (MDR2/3, ABCB4) for efflux of phosphatidylcholine; and a multidrug resistance-associated protein 2 (MRP2, ABCC2), which effluxes glucuronidated and sulfated bile acids. ${ }^{6}$ In the terminal ileum, conjugated bile acids are reabsorbed by an apical sodium-dependent bile salt transporter (ASBT) located in the brush border membrane of the enterocytes. ${ }^{4}$ This sodium- and potential-driven transporter moves bile acids from the lumen of the small intestine across the apical brush border membrane. Bile acids are then shuttled to the basolateral membrane and effluxed into the portal circulation by organic solute transporter $\alpha$ and $\beta$ (OST $\alpha$-OST $\beta$ ). ${ }^{16}$ The expression of OST $\alpha$ -OST $\beta$ is positively regulated by bile acids and FXR. ${ }^{12}$ Bile acids secreted from the intestine are uptaken predominantly by a sinusoidal $\mathrm{Na}^{+}$-dependent taurocholate cotransport peptide (NTCP) into hepatocytes. ${ }^{16}$ It is known that NTCP gene expression is suppressed by high levels of bile acids as an adaptive response to reduce their entry into the hepatocytes. Bile acids affect NTCP gene transcription via the activation of FXR. ${ }^{16}$ FXR does not interact directly with the NTCP promoter, but it induces the expression of other factors, namely SHP, retinoic acid receptor, retinoid X receptor RAR/RXR heterodimer, and HNF1 $\alpha$, to indirectly repress NTCP expression. ${ }^{16}$

Apart from its role in bile acid homeostasis, FXR has been implicated in the regulation of lipid homeostasis. It was reported that FXR null mice have increased hepatic bile acids, cholesterol, triglycerides, and proatherogenic serum lipoprotein profiles, as well as decreased bile acid pools and fecal bile excretion with decreased expression of the major hepatic canalicular bile acid transport proteins. ${ }^{17}$ In con- 
trast, administration of a synthetic FXR agonist GW4064 reduces plasma triglyceride, cholesterol, and free fatty acid levels in diabetic $d b / d b$ mice and wild-type mice, but not FXR $/$ mice. ${ }^{18}$ Several mechanisms have been proposed to explain the regulation of FXR in triglyceride and cholesterol metabolism. 1) Bile acid-activated FXR suppresses hepatic de novo lipogenesis through inhibition of sterolregulatory element-binding protein 1 (SREBP-1c), a master transcription factor that controls lipogenic gene expression, including that of acetyl CoA synthetase, acetyl CoA carboxylase, stearoyl CoA desaturase 1 , and fatty acid synthase, in the SHP-dependent pathway. ${ }^{19}$ However, a triglyceride-lowering effect of bile acid or FXR agonists through the FXR-SHP-SREBP1c pathway remains somewhat controversial because $\mathrm{CA}$ treatment in $\mathrm{SHP}^{-} /$mice still showed a decrease in hepatic triglyceride accumulation in some studies, implying that other pathways may also be involved independent of the FXRSHP-SREBP1c pathway. ${ }^{20}$ 2) FXR activated by bile acid or a synthetic FXR agonist also upregulates peroxisome proliferator-activated receptor alpha (PPAR $\alpha$ ) and its target gene, carnitine palmitoyltransferase, resulting in stimulation of fatty acid $\beta$-oxidation in humans, but not in mice. ${ }^{21}$ 3) FXR regulates expression of genes involved in triglyceride metabolism and plasma lipoprotein clearance, including the very-low-density lipoprotein (VLDL) receptor and apolipoprotein (Apo) C-II and ApoA-V, which are the activators of lipoprotein lipase (LPL); LPL is involved in lipolysis of triglycerides from triglyceride-rich lipoproteins, VLDL and chylomicron; ApoC-III and angiopoietin-like protein-3, which inhibit LPL activity; and syndecan-1, which is known to be responsible for uptake and clearance of remnant particles via its heparan sulfate-dependent pathway. ${ }^{4}$ ) FXR activation by CA and FXR agonists showed a reduction in blood cholesterol levels, mainly due to a decrease in circulating high-density lipoprotein (HDL), by which FXR activation may influence reverse cholesterol transport machinery, a system to move excess cholesterol from peripheral tissues back to the liver through up-regulation of scavenger receptor class B type I (SR-BI), the HDL receptor, and cholesteryl ester transfer protein expression and by decreasing expression of ApoA1, the major protein constituent of HDL. ${ }^{22}$

In addition, many recent studies have implicated FXR functions in the regulation of glucose metabolism. Several studies have reported that bile acids inhibit the expression of genes involved in gluconeogenesis, including phosphoenolpyruvate carboxykinase, glucose6-phosphatase, and fructose 1,6-phosphatase, in part through SHP- dependent inhibition of HNF4 $\alpha$ and FoxO1. ${ }^{23,24}$ In the study of Zhang et al. ${ }^{18}$, activation of FXR by the synthetic agonist GW4064 or by hepatic overexpression of constitutively active FXR by adenovirus-mediated gene transfer significantly lowered blood glucose levels in both diabetic $d b / d b$ and wild-type mice, in accordance with repressed hepatic gluconeogenic genes and increased hepatic glycogen synthesis, glycogen content, and enhanced insulin sensitivity. However, FXR null mice showed glucose intolerance and decreased insulin sensitivity with impaired hepatic insulin signaling, which was improved by FXR overexpression mediated by adenovirus in $\mathrm{FXR}^{-}{ }^{-}$mice, but not by GW4064 treatment. ${ }^{18}$ Furthermore, bile acid activation of FXR stimulates the insulin/AKT pathway, which phosphorylates and inactivates GSK3 $\beta$, leading to stimulation of glycogen synthesis. ${ }^{25}$ In addition, FXR and its agonist, CDCA, may control the expression of glucose transporter 4 and thereby affect systemic glucose homeostasis. ${ }^{26}$ This favorable effect on glucose metabolism may be mediated, in part, by induction of FGF15 (human ortholog, FGF19) in the enterocytes, which activates a hepatic FGFR4 signal. FGF15/19 stimulates hepatic protein and glycogen synthesis through the activation of the ERK1/2 pathway and represses gluconeogenesis through inactivation of the cAMP regulatory element-binding protein (CREB)-peroxisome proliferator-activated receptor $\gamma$ coactivator-1 $\alpha$ (PGC- $1 \alpha)$ pathway. ${ }^{27,28}$ However, some studies have reported the failure of $\mathrm{FXR}^{-} /$ mice to develop overt diabetes, undesignable effects of the FXR agonist GW4064-induced energy expenditure attenuation in high fat diet-induced obese mice, and hepatocellular carcinoma development in FGF19 transgenic mice. ${ }^{12,29,30}$ Therefore, the role of FXR in the regulation of glucose metabolism needs further investigation.

FXR is also known to be important in inflammation, which plays an essential role in the pathogeneses of NAFLD and NASH. FXR activation strongly suppressed the expression of genes involved in inflammation, such as interleukin 6 (IL-6), suppressing C-reactive protein production, and nuclear factor $\kappa \mathrm{B}(\mathrm{NF}-\kappa \mathrm{B})$, in the liver. ${ }^{20}$ In the liver, the molecular mechanism by which FXR antagonizes inflammation may be due to an acetyl/small ubiquitin-like modifier (SUMO) switch, such that SUMOylation of FXR increased its interaction with NF- $\kappa \mathrm{B}$, which suppresses NF- $\kappa \mathrm{B}$-mediated transcriptional induction of inflammatory genes. ${ }^{31}$ However, as the role of FXR in inflammation has also been reported to be controversial ${ }^{4}$, it remains to be determined how FXR modulates inflammation and immune function. 


\section{FXR agonists for the treatment of NAFLD/NASH}

Activation of FXR has shown beneficial effects on lipid and glucose homeostasis, as well as the inflammatory response. Several preclinical studies using experimental models of human liver disease have demonstrated the efficacy of FXR agonists as potential therapeutic agents in NASH and NAFLD. A synthetic bile acid derivative, obeticholic acid (OCA, 6a-ethyl CDCA or INT-747), is the first-inclass FXR agonist to be tested in clinical trials. It was first described in 2002 as a potent and selective steroidal FXR agonist. ${ }^{32}$ In animal studies, OCA reverses insulin resistance and hepatic steatosis, inhibits gluconeogenesis and lipogenesis, and also shows anti-inflammatory and anti-fibrotic activities. ${ }^{33,34}$ Recently, the effect of OCA on insulin sensitivity has been investigated in a randomized, placebo-controlled clinical trial of patients with NAFLD and type 2 diabetes. ${ }^{35}$ In this clinical trial, the administration of 25 or $50 \mathrm{mg}$ OCA for six weeks improved insulin resistance and reduced markers of liver inflammation and fibrosis. ${ }^{35}$ More recently, the Phase IIb FLINT (FXR Lignad Obeticholic Acid in NASH Treatment) trial, which included NASH patients treated with $25 \mathrm{mg}$ OCA for 72 weeks, provided information on the metabolic effects of FXR agonism on glucose and lipid metabolism in humans. ${ }^{36}$ In that multicenter, double-blinded, placebo-controlled clinical trial, patients treated with OCA showed improved histological features of NASH (i.e., improvement in NAFLD Activity Score, inflammation, and fibrosis), as well as reduced serum liver enzyme levels. In addition, unexpectedly, increased insulin serum levels and a higher homeostasis model assessment (HOMA) index were found in the OCA treatment group. ${ }^{36} \mathrm{~Pa}-$ tients who received OCA also showed significant changes in serum lipids, consisting of an increase in total serum cholesterol and LDLcholesterol levels and a modest, but significant, reduction in HDLcholesterol, which are likely secondary to increased FGF19 production, acting to suppress hepatic bile acid synthesis and hepatic demand for cholesterol. ${ }^{36}$ Pruritus, a known side effect of OCA, was more frequently observed in the OCA-treated group. ${ }^{36}$ These results suggest that, in spite of some serious concerns about cholesterol elevation and increased diabetic risk, OCA might be beneficial in preventing progression of NASH, though long-term studies are needed to explore the clinical relevance of FXR ligand treatment-related dyslipidemia. $^{37}$
In addition to OCA, other FXR agonists are being investigated in preclinical studies and ongoing clinical trials. GW4064, developed by Maloney et $\mathrm{al}^{38}$, is a potent and selective nonsteroidal FXR agonist, decreases serum triglycerides, and improves insulin sensitivity and glucose tolerance in rodents. ${ }^{18}$ Since then, many pharmaceutical companies have used GW4064 as a structural template for their efforts in identifying novel patentable FXR agonists. ${ }^{39}$ WAY-362450 (FXR-450 or XL335), developed by Flatt et al. $^{40}$, is also a potent and selective FXR agonist; administration of WAY-362450 significantly lowered serum triglyceride and total cholesterol levels and inhibited aortic atherosclerotic formation in a dose-dependent manner in mice. ${ }^{40}$ PX-102/PX-104 is a fully-synthetic, potent nonsteroidal FXR agonist currently being evaluated in a phase IIa randomized, clinical trial of patients with NAFLD. ${ }^{39}$

\section{Implications of gut microbiota for bile acid-FXR signaling in NAFLD}

The gut microbiota is thought to significantly influence metabolism and energy expenditure and is associated with the development of metabolic diseases, such as obesity, type 2 diabetes mellitus, and insulin resistance. Both NAFLD and NASH are strongly linked to metabolic disease; accordingly, roles of gut microbiota in the etiology of NAFLD/NASH have been largely studied in mice and humans. ${ }^{41}$ It has been reported that obese humans and mice showed a higher abundance of phylum Firmicutes, which is a short-chain fatty acid (e.g., acetate, butyrate, and propionate)-producing bacteria that can subsequently enhance energy absorption to accelerate obesity-associated NAFLD. ${ }^{42,43}$ Spencer et al. ${ }^{44}$ observed the changes in gut microbiome composition during development of fatty liver with choline-deficiency in humans. Several human studies have examined the differences in the gut microbiome among cohorts of individuals with NAFLD and NASH. ${ }^{44-48}$ However, due to the great variability in study design, methods, and clinical endpoints, it remains challenging to interpret the clinical significance of intestinal dysbiosis in human NAFLD. ${ }^{49}$ Furthermore, recent studies of gut bacteria transplantation have shown a direct relationship between gut microbiome and metabolic disease, including obesity and NAFLD. It was reported that, when lean germ-free mice were treated with the fecal microbiota from obese mice, there was an increase in insulin resistance and hepatic steatosis with increased levels of phylum Firmicutes. ${ }^{50}$ 
When bacteria from lean humans were transferred to germ-free mice, the mice became resistant to obesity, whereas mice receiving bacteria from obese humans were predisposed to obesity. ${ }^{51}$

Several possible mechanisms by which gut microbiota and their metabolites could influence NALFD have been suggested, including its effects on the digestion and absorption of nutrients, the regulation of the host immune system such as activation of endotoxin receptors, Toll-like-receptor (TLR)-mediated signaling, and the production of gut hormones such as glucagon-like peptide 1 (GLP-1), which subsequently leads to an effect on the overall metabolism of the host. ${ }^{45} \mathrm{Ad}-$ ditionally, it is well established that the gut microbiota has profound effects on bile acid metabolism by promoting enzymatic activity (e.g., deconjugation, dehydrogenation, and dehydroxylation of primary bile acids) in the distal small intestine and colon, thus regulating bile acid pool size and composition. ${ }^{52}$ Interestingly, it is reported that germ-free mice and mice lacking intestinal FXR are resistant to high-fat diet-induced obesity and hepatic fat accumulation, suggesting that gut microbiota promote weight gain and hepatic steatosis through FXR-dependent mechanisms. ${ }^{2}$ Of note, in mice, taurineconjugated MCAs (T-MCAs, TaMCA, and T $\beta M C A$ ) were identified as FXR antagonists, which significantly reduce the bile acid-induced expression of FGF15 in the ileum, followed by upregulation of CYP7A1 and bile acid synthesis in the liver. In contrary, the conversion of T-MCA to MCA by gut microbiota alleviated T-MCA-induced suppression of FXR signaling. ${ }^{52}$ It has also been reported that modulation of the mouse gut microbiota using antibiotics or the antioxidant tempol increases the level of T $\beta M C A$ and suppresses intestinal FXR activity, followed by decreased ceramide production, which downregulates hepatic SREBP1c and decreases de novo lipogenesis in the liver. ${ }^{2,53}$ These findings suggest that gut microbiota regulate liver lipid metabolism by modulating intestinal FXR signaling. Therapeutic interventions targeting gut microbiota, such as nutraceutical (diet, probiotics), pharmaceutical (antibiotic, tempol), or surgical (bariatric surgery) interventions, in NAFLD/NASH may act favorably through the induction of changes in host bile acid profiles and influence FXR signaling. ${ }^{54}$ Meanwhile, it has been reported that oral treatment of glycine- $\beta$-muricholic acid (Gly-MCA), a derivative generated by in-silico modeling of T $\beta M C A$ binding to FXR, in mice on a high-fat diet inhibited FXR signaling in the ileum and decreased obesity, insulin resistance, and fatty liver, similar to the effects observed in both tempol- and antibiotic-treated mice, while he- patic FXR signaling was not affected. This is of notable importance because the inhibition of hepatic FXR could result in hepatic steatosis and toxicity, as seen in global FXR knockout and liver-specific FXR knockout mice. ${ }^{2,17,53,55}$ A more recent study has demonstrated that the inhibition of intestinal FXR signals by an endogenous bile acid T $\beta M C A$ or its derivative results in improved metabolic disturbance, including high fat diet-induced obesity, insulin resistance, and NAFLD, suggesting that this pathway could be a novel mechanism to treat metabolic disease. ${ }^{56}$ Although of mechanistic interest, studies in rodents provide limited information on bile metabolism in humans, because human and mouse bile acids markedly differ; humans have primarily CA, CDCA, and glycine conjugates, while mice make MCA and primarily taurine conjugates. Therefore, in humans, it is unclear whether other bile acid conjugates function to antagonize intestinal FXR activity, as occurs with TßMCA in mice. Additional studies are needed to investigate whether these findings can be translated to humans and other species. ${ }^{56}$

\section{Conclusion}

In this review, evidence indicates that bile acid signaling plays a central role in the pathogenesis of several metabolic diseases, including obesity and NAFLD/NASH. Also, FXR affects several metabolic pathways through its specific target genes, regulating bile acid synthesis and homeostasis and glucose and lipid metabolism. Therefore, specific targeting of bile acid-related pathways, including the FXR pathway, appears promising, though there are still many challenges, including selectivity between FXR and other bile acid receptors, tissue-specific activity of drug candidates, and safety issues, particularly those related to the long-term use of a given agent.

\section{Conflicts of Interest}

The authors have no conflicts of interest to declare.

\section{Acknowledgments}

This study was supported by Samsung Biomedical Research Institute grant. 


\section{References}

1. Ahmed A, Wong RJ, Harrison SA. Nonalcoholic fatty liver disease review: diagnosis, treatment, and outcomes. Clin Gastroenterol Hepatol 2015;13:2062-70.

2. Jiang C, Xie C, Li F, Zhang L, Nichols RG, Krausz KW, et al. Intestinal farnesoid X receptor signaling promotes nonalcoholic fatty liver disease. J Clin Invest 2015;125:386-402.

3. Insull W Jr. Clinical utility of bile acid sequestrants in the treatment of dyslipidemia: a scientific review. South Med J 2006;99:25773.

4. Chiang JY. Bile acid metabolism and signaling. Compr Physiol 2013;3:1191-212.

5. Pircher PC, Kitto JL, Petrowski ML, Tangirala RK, Bischoff ED, Schulman IG, et al. Farnesoid X receptor regulates bile acid-amino acid conjugation. J Biol Chem 2003;278:27703-11.

6. Qi Y, Jiang C, Cheng J, Krausz KW, Li T, Ferrell JM, et al. Bile acid signaling in lipid metabolism: metabolomic and lipidomic analysis of lipid and bile acid markers linked to anti-obesity and anti-diabetes in mice. Biochim Biophys Acta 2015;1851:19-29.

7. Reihnér E, Björkhem I, Angelin B, Ewerth S, Einarsson K. Bile acid synthesis in humans: regulation of hepatic microsomal cholesterol 7 alpha-hydroxylase activity. Gastroenterology 1989;97: 1498-505.

8. Angelin B, Einarsson K, Hellström K, Leijd B. Effects of cholestyramine and chenodeoxycholic acid on the metabolism of endogenous triglyceride in hyperlipoproteinemia. J Lipid Res 1978;19: 1017-24.

9. Makishima M, Okamoto AY, Repa JJ, Tu H, Learned RM, Luk A, et al. Identification of a nuclear receptor for bile acids. Science 1999;284:1362-5.

10. Parks DJ, Blanchard SG, Bledsoe RK, Chandra G, Consler TG, Kliewer SA, et al. Bile acids: Natural ligands for an orphan nuclear receptor. Science 1999;284:1365-8.

11. Wang H, Chen J, Hollister K, Sowers LC, Forman BM. Endogenous bile acids are ligands for the nuclear receptor FXR/BAR. Mol Cell 1999;3:543-53.

12. Schaap FG, Trauner M, Jansen PL. Bile acid receptors as targets for drug development. Nat Rev Gastroenterol Hepatol 2014;11:5567.

13. Forman BM, Goode E, Chen J, Oro AE, Bradley DJ, Perlmann T, et al. Identification of a nuclear receptor that is activated by farnesol metabolites. Cell 1995;81:687-93.

14. Seol W, Choi HS, Moore DD. Isolation of proteins that interact specifically with the retinoid $\mathrm{X}$ receptor: two novel orphan receptors. Mol Endocrinol 1995;9:72-85.

15. Lefebvre P, Cariou B, Lien F, Kuipers F, Staels B. Role of bile acids and bile acid receptors in metabolic regulation. Physiol Rev 2009; 89:147-91.

16. Dawson PA, Lan T, Rao A. Bile acid transporters. J Lipid Res 2009;50:2340-57.

17. Sinal CJ, Tohkin M, Miyata M, Ward JM, Lambert G, Gonzalez FJ. Targeted disruption of the nuclear receptor FXR/BAR impairs bile acid and lipid homeostasis. Cell 2000;102:731-44.

18. Zhang Y, Lee FY, Barrera G, Lee H, Vales C, Gonzalez FJ, et al. Activation of the nuclear receptor FXR improves hyperglycemia and hyperlipidemia in diabetic mice. Proc Natl Acad Sci U S A 2006;103:1006-11.

19. Watanabe M, Houten SM, Wang L, Moschetta A, Mangelsdorf DJ, Heyman RA, et al. Bile acids lower triglyceride levels via a pathway involving FXR, SHP, and SREBP-1c. J Clin Invest 2004;113: 1408-18.

20. Li Y, Jadhav K, Zhang Y. Bile acid receptors in non-alcoholic fatty liver disease. Biochem Pharmacol 2013;86:1517-24.

21. Pineda Torra I, Claudel T, Duval C, Kosykh V, Fruchart JC, Staels B. Bile acids induce the expression of the human peroxisome proliferator-activated receptor alpha gene via activation of the farnesoid X receptor. Mol Endocrinol 2003;17:259-72.

22. Lambert G, Amar MJ, Guo G, Brewer HB Jr, Gonzalez FJ, Sinal CJ. The farnesoid X-receptor is an essential regulator of cholesterol homeostasis. J Biol Chem 2003;278:2563-70.

23. De Fabiani E, Mitro N, Gilardi F, Caruso D, Galli G, Crestani M. Coordinated control of cholesterol catabolism to bile acids and of gluconeogenesis via a novel mechanism of transcription regulation linked to the fasted-to-fed cycle. J Biol Chem 2003;278: 39124-32.

24. Yamagata K, Daitoku H, Shimamoto Y, Matsuzaki H, Hirota K, Ishida J, et al. Bile acids regulate gluconeogenic gene expression via small heterodimer partner-mediated repression of hepatocyte nuclear factor 4 and Foxo1. J Biol Chem 2004;279:23158-65.

25. Li T, Owsley E, Matozel M, Hsu P, Novak CM, Chiang JY. Transgenic expression of cholesterol 7alpha-hydroxylase in the liver 
prevents high-fat diet-induced obesity and insulin resistance in mice. Hepatology 2010;52:678-90.

26. Shen H, Zhang Y, Ding H, Wang X, Chen L, Jiang H, et al. Farnesoid X receptor induces GLUT4 expression through FXR response element in the GLUT4 promoter. Cell Physiol Biochem 2008;22:114.

27. Kir S, Beddow SA, Samuel VT, Miller P, Previs SF, Suino-Powell K, et al. FGF19 as a postprandial, insulin-independent activator of hepatic protein and glycogen synthesis. Science 2011;331:1621-4.

28. Potthoff MJ, Boney-Montoya J, Choi M, He T, Sunny NE, Satapati S, et al. FGF15/19 regulates hepatic glucose metabolism by inhibiting the CREB-PGC-1 $\alpha$ pathway. Cell Metab 2011;13:729-38.

29. Prawitt J, Abdelkarim M, Stroeve JH, Popescu I, Duez H, Velagapudi VR, et al. Farnesoid X receptor deficiency improves glucose homeostasis in mouse models of obesity. Diabetes 2011;60:186171.

30. Watanabe M, Horai Y, Houten SM, Morimoto K, Sugizaki T, Arita E, et al. Lowering bile acid pool size with a synthetic farnesoid X receptor (FXR) agonist induces obesity and diabetes through reduced energy expenditure. J Biol Chem 2011;286:26913-20.

31. Kim DH, Xiao Z, Kwon S, Sun X, Ryerson D, Tkac D, et al. A dysregulated acetyl/SUMO switch of FXR promotes hepatic inflammation in obesity. EMBO J 2015;34:184-99.

32. Pellicciari R, Fiorucci S, Camaioni E, Clerici C, Costantino G, Maloney PR, et al. 6alpha-ethyl-chenodeoxycholic acid (6-ECD$\mathrm{CA})$, a potent and selective FXR agonist endowed with anticholestatic activity. J Med Chem 2002;45:3569-72.

33. Cipriani S, Mencarelli A, Palladino G, Fiorucci S. FXR activation reverses insulin resistance and lipid abnormalities and protects against liver steatosis in Zucker (fa/fa) obese rats. J Lipid Res 2010; 51:771-84.

34. Mencarelli A, Renga B, Migliorati M, Cipriani S, Distrutti E, Santucci L, et al. The bile acid sensor farnesoid X receptor is a modulator of liver immunity in a rodent model of acute hepatitis. J Immunol 2009;183:6657-66.

35. Mudaliar S, Henry RR, Sanyal AJ, Morrow L, Marschall HU, Kipnes $\mathrm{M}$, et al. Efficacy and safety of the farnesoid X receptor agonist obeticholic acid in patients with type 2 diabetes and nonalcoholic fatty liver disease. Gastroenterology 2013;145:574-82.

36. Neuschwander-Tetri BA, Loomba R, Sanyal AJ, Lavine JE, Van Natta ML, Abdelmalek MF, et al. Farnesoid X nuclear receptor li- gand obeticholic acid for non-cirrhotic, non-alcoholic steatohepatitis (FLINT): a multicentre, randomised, placebo-controlled trial. Lancet 2015;385:956-65.

37. Ali AH, Carey EJ, Lindor KD. Recent advances in the development of farnesoid X receptor agonists. Ann Transl Med 2015;3:5.

38. Maloney PR, Parks DJ, Haffner CD, Fivush AM, Chandra G, Plunket $\mathrm{KD}$, et al. Identification of a chemical tool for the orphan nuclear receptor FXR. J Med Chem 2000;43:2971-4.

39. Gege C, Kinzel O, Steeneck C, Schulz A, Kremoser C. Knocking on FXR's door: the "hammerhead"-structure series of FXR agonists - amphiphilic isoxazoles with potent in vitro and in vivo activities. Curr Top Med Chem 2014;14:2143-58.

40. Flatt B, Martin R, Wang TL, Mahaney P, Murphy B, Gu XH, et al. Discovery of XL335 (WAY-362450), a highly potent, selective, and orally active agonist of the farnesoid X receptor (FXR). J Med Chem 2009;52:904-7.

41. He X, Ji G, Jia W, Li H. Gut microbiota and nonalcoholic fatty liver disease: insights on mechanism and application of metabolomics. Int J Mol Sci 2016;17:300.

42. Ley RE, Turnbaugh PJ, Klein S, Gordon JI. Microbial ecology: human gut microbes associated with obesity. Nature 2006;444:10223.

43. Turnbaugh PJ, Ley RE, Mahowald MA, Magrini V, Mardis ER, Gordon JI. An obesity-associated gut microbiome with increased capacity for energy harvest. Nature 2006;444:1027-31.

44. Spencer MD, Hamp TJ, Reid RW, Fischer LM, Zeisel SH, Fodor AA. Association between composition of the human gastrointestinal microbiome and development of fatty liver with choline deficiency. Gastroenterology 2011;140:976-86.

45. Zhu L, Baker SS, Gill C, Liu W, Alkhouri R, Baker RD, et al. Characterization of gut microbiomes in nonalcoholic steatohepatitis (NASH) patients: a connection between endogenous alcohol and NASH. Hepatology 2013;57:601-9.

46. Mouzaki M, Comelli EM, Arendt BM, Bonengel J, Fung SK, Fischer SE, et al. Intestinal microbiota in patients with nonalcoholic fatty liver disease. Hepatology 2013;58:120-7.

47. Raman M, Ahmed I, Gillevet PM, Probert CS, Ratcliffe NM, Smith S, et al. Fecal microbiome and volatile organic compound metabolome in obese humans with nonalcoholic fatty liver disease. Clin Gastroenterol Hepatol 2013;11:868-75.

48. Wong VW, Tse CH, Lam TT, Wong GL, Chim AM, Chu WC, et 
al. Molecular characterization of the fecal microbiota in patients with nonalcoholic steatohepatitis - a longitudinal study. PLoS One 2013;8:e62885.

49. Wieland A, Frank DN, Harnke B, Bambha K. Systematic review: microbial dysbiosis and nonalcoholic fatty liver disease. Aliment Pharmacol Ther 2015;42:1051-63.

50. Le Roy T, Llopis M, Lepage P, Bruneau A, Rabot S, Bevilacqua C, et al. Intestinal microbiota determines development of non-alcoholic fatty liver disease in mice. Gut 2013;62:1787-94.

51. Ridaura VK, Faith JJ, Rey FE, Cheng J, Duncan AE, Kau AL, et al. Gut microbiota from twins discordant for obesity modulate metabolism in mice. Science 2013;341:1241214.

52. Sayin SI, Wahlström A, Felin J, Jäntti S, Marschall HU, Bamberg K, et al. Gut microbiota regulates bile acid metabolism by reducing the levels of tauro-beta-muricholic acid, a naturally occurring FXR antagonist. Cell Metab 2013;17:225-35.

53. Li F, Jiang C, Krausz KW, Li Y, Albert I, Hao H, et al. Microbiome remodelling leads to inhibition of intestinal farnesoid $\mathrm{X}$ receptor signalling and decreased obesity. Nat Commun 2013;4:2384.

54. Arab JP, Karpen SJ, Dawson PA, Arrese M, Trauner M. Bile acids and nonalcoholic fatty liver disease: Molecular insights and therapeutic perspectives. Hepatology 2016 [Epub ahead of print].

55. Jiang C, Xie C, Lv Y, Li J, Krausz KW, Shi J, et al. Intestine-selective farnesoid $\mathrm{X}$ receptor inhibition improves obesity-related metabolic dysfunction. Nat Commun 2015;6:10166.

56. Gonzalez FJ, Jiang C, Patterson AD. An intestinal microbiotafarnesoid X receptor axis modulates metabolic disease. Gastroenterology 2016 [Epub ahead of print]. 